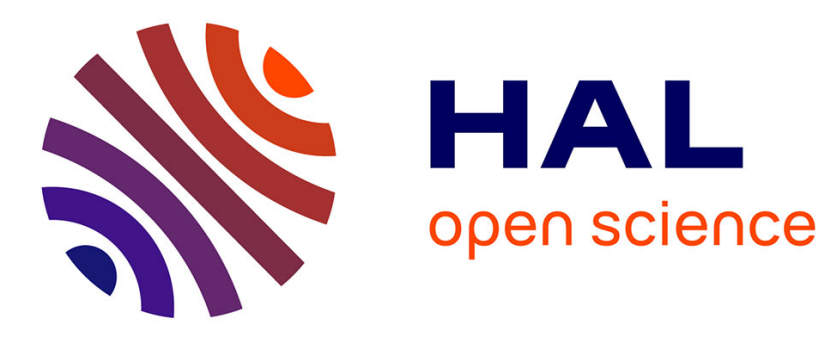

\title{
Image-based Gender Estimation from Body and Face across Distances
}

Ester Gonzalez-Sosa, Antitza Dantcheva, Ruben Vera-Rodriguez, Jean-Luc Dugelay, François Brémond, Julian Fierrez

\section{- To cite this version:}

Ester Gonzalez-Sosa, Antitza Dantcheva, Ruben Vera-Rodriguez, Jean-Luc Dugelay, François Brémond, et al.. Image-based Gender Estimation from Body and Face across Distances. 23rd International Conference on Pattern Recognition (ICPR 2016): "Image analysis and machine learning for scene understanding", Dec 2016, Cancun, Mexico. hal-01384324

\section{HAL Id: hal-01384324 \\ https://hal.science/hal-01384324}

Submitted on 19 Oct 2016

HAL is a multi-disciplinary open access archive for the deposit and dissemination of scientific research documents, whether they are published or not. The documents may come from teaching and research institutions in France or abroad, or from public or private research centers.
L'archive ouverte pluridisciplinaire HAL, est destinée au dépôt et à la diffusion de documents scientifiques de niveau recherche, publiés ou non, émanant des établissements d'enseignement et de recherche français ou étrangers, des laboratoires publics ou privés. 


\title{
Image-based Gender Estimation from Body and Face across Distances
}

\author{
Ester Gonzalez-Sosa*, Antitza Dantcheva ${ }^{\dagger}$, Ruben Vera-Rodriguez ${ }^{*}$ \\ Jean-Luc Dugelay ${ }^{\ddagger}$, François Brémond ${ }^{\dagger}$ and Julian Fierrez* \\ *Biometric Recognition Group - ATVS, EPS, Universidad Autonoma de Madrid \\ \{ester.gonzalezs, ruben.vera, julian.fierrez\}@uam.es \\ ${ }^{\dagger}$ INRIA, Sophia Antipolis, \{antitza.dantcheva, francois.bremond\}einria.fr

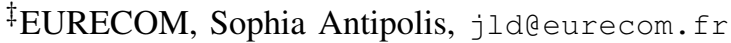

\begin{abstract}
Gender estimation has received increased attention due to its use in a number of pertinent security and commercial applications. Automated gender estimation algorithms are mainly based on extracting representative features from face images. In this work we study gender estimation based on information deduced jointly from face and body, extracted from single-shot images. The approach addresses challenging settings such as lowresolution-images, as well as settings when faces are occluded. Specifically the face-based features include local binary patterns (LBP) and scale-invariant feature transform (SIFT) features, projected into a PCA space. The features of the novel bodybased algorithm proposed in this work include continuous shape information extracted from body silhouettes and texture information retained by HOG descriptors. Support Vector Machines (SVMs) are used for classification for body and face features. We conduct experiments on images extracted from video-sequences of the Multi-Biometric Tunnel database, emphasizing on three distance-settings: close, medium and far, ranging from full body exposure (far setting) to head and shoulders exposure (close setting). The experiments suggest that while face-based gender estimation performs best in the close-distance-setting, body-based gender estimation performs best when a large part of the body is visible. Finally we present two score-level-fusion schemes of face and body-based features, outperforming the two individual modalities in most cases.
\end{abstract}

\section{INTRODUCTION}

Soft biometrics are physical, behavioural or adhered ancillary attributes that can be inferred from subjects in addition to classical / primary biometrics [10], [31]. Related traits include gender, age, ethnicity, body height, and eye color among others. Despite the limited distinctiveness, as opposed to primary biometrics, such traits have gained high pertinence due to the plethora of benefits they encompass, such as the ability to bridge the gap between human and machine in the context of person recognition [39].

Gender estimation is a soft biometric trait, that has specifically received increased attention for its use in surveillance applications [33], human computer interaction, anonymous customized advertisement systems [30], and image retrieval systems [28]. In the area of biometrics, gender estimation can increase the reliability of a classical biometric system [19], as well as reduce efficiently the search space for a primary biometric [12], [11].

The majority of automated gender estimation algorithms have been designed for face images (see Section I-A). We note though, that in applications including video surveillance and anonymous customized advertisement systems, image or video-data often shows the face, as well as the body of a subject. Limited attention has been given to the joint exploitation of face and body for gender estimation, with only few works analyzing jointly gait and face [35], [41]. One challenge in such a multimodal approach is the requirement of two acquisition cameras: one frontal (capturing the face) and one lateral (capturing the gait), bringing to the fore the need for camera-synchronization. In addition, capturing a full gait cycle (a sequence of several images) without occlusions (from persons, obstacles, etc.) is rather challenging in practical scenarios.

Deviating from face and gait approaches, in this work we propose a novel approach that extracts face and body features from one single shot image, achieving promising gender estimation results. The utilized body gender estimation algorithm is novel and extracts geometric and texture features from single-shot silhouettes. We provide analysis of performance of the body and face algorithms vs. the distance-between-subjectand-camera. In this context we define three distance-settings: far, medium and close, where the visibility and resolution of face and body improve / degrade for the three settings. We chose the three distance-settings as representative examples for varying subject-to-camera-distances. Finally we also present fusion of the face and body gender estimation algorithms, predominantly outperforming the individual algorithms.

The rest of the paper is organized as follows. Relevant literature on gender estimation is reviewed in Section I. Section II presents the utilized face and proposed body gender estimation algorithms, a brief description of the used Tunnel database [34] is given in Section III and the related results are summarized in Section IV. Conclusions on the effectiveness of the proposed multimodal gender estimation system are drawn in Section V.

\section{A. Gender from Face}

Classical feature-based approaches extract discriminative facial features and analyse these towards determining the gender of a person. The challenge is inherited by the fact that female and male average faces are very similar [22]. Further challenges are faced in unconstrained settings where 


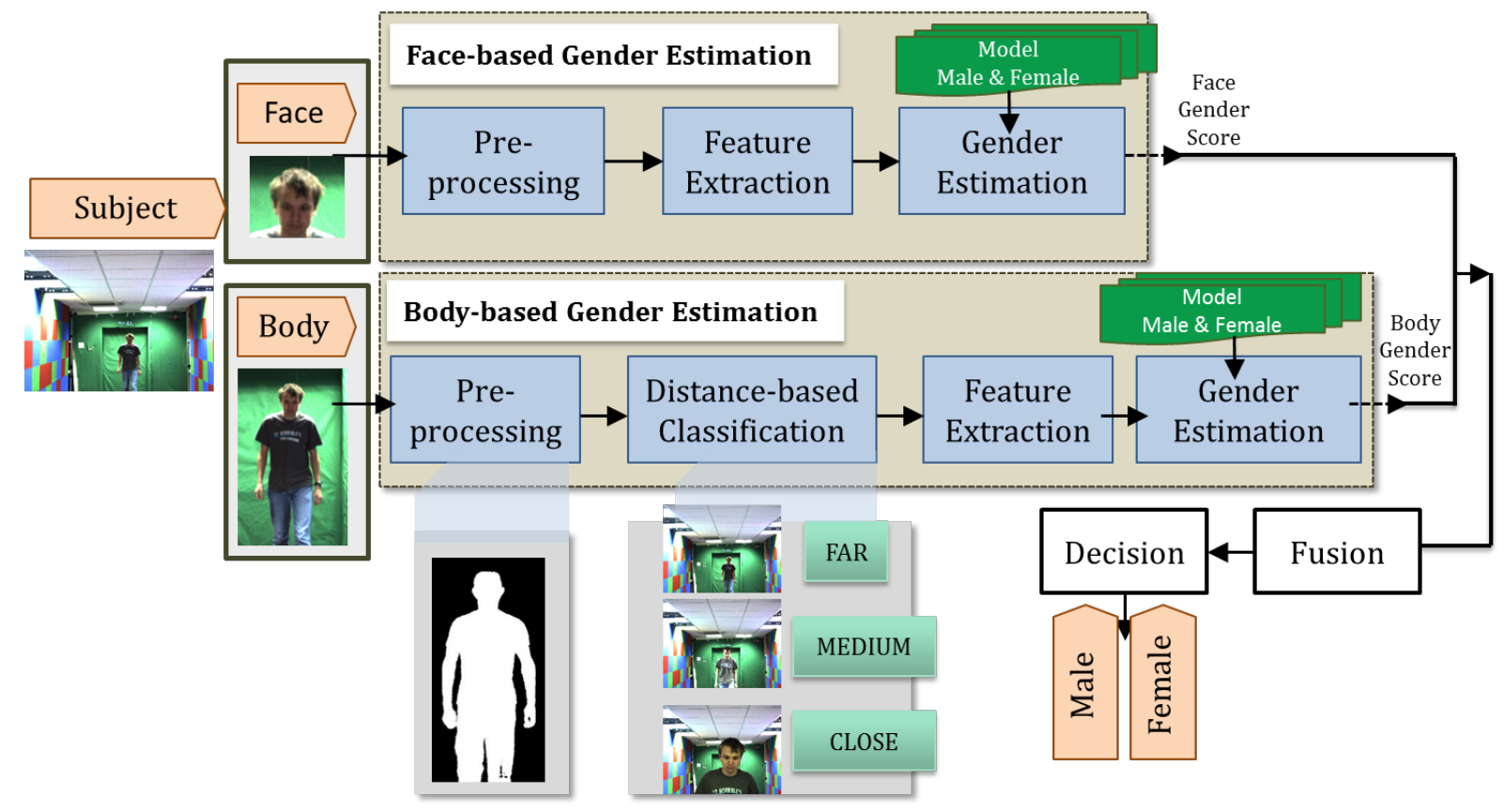

Fig. 1. Fusion of face and body information for gender estimation. Given a subject, gender is estimated through a state-of-the-art face-based algorithm (OpenBR). We propose to additionally estimate gender through body information to improve the performance in challenging settings such as low-resolutionimages or occluded faces. Face and body gender estimates are combined to give an improved estimation of gender in the three specific distance-settings.

illumination, facial expressions and ethnicity can be confound covariates. While gender estimation accuracy of up to $99.3 \%$ is achieved in highly constrained settings, this performance is substantially decreased in unconstrained settings. Gender estimation algorithms are widely reviewed in $\mathrm{Ng}$ et al. [29], Bekios-Calfa et al. [2], and Dantcheva et al. [10].

The majority of face gender estimation algorithms contain two steps following face detection, namely feature extraction and classification. In the context of feature extraction SIFT [38], LBP [26], semi-supervised discriminant analysis (SDA) [3] or combinations of different features [40] have been studied. A review of classification methods used for gender estimation [26] concluded that the best classification rates were achieved by SVM.

\section{B. Gender from Body}

The human body contains some cues such as height, width, waist, hips, chest, shoulder, etc, that may help humans as well as automated systems to determine gender. Currie and Little [8] give empirical evidence on the sexual dimorphism of such body measures.

Cao et al. were the first to develop an algorithm predicting gender from the body [5], based on full body-silhouette (frontal and back) represented by histogram of oriented gradients (HOG). Later Collins et al. [7] proposed the use of body-appearance and shape, respectively represented by pyramid histogram of words and pyramid HOG, respectively (only frontal). Guo et al. [18] firstly classified the body pose (frontal, back or mixed) and estimated gender based on a view-dependent algorithm using biologically-inspired features
(BIF). The predominant classifier for body, as well as face gender estimation has been SVM.

Latest works predict gender based on RGB-D (depth) data [25] or learn features from deep learning techniques (from front and from back) [1].

\section{Gender from other traits}

We note that gender has been predominantly estimated from facial images [10]. Other modalities that have been studied in this context include gait [36], facial smiling behavior [4], [9], or speech [37]. In addition, there are some hybrid approaches fusing algorithms based on different biometric modalities such as face and gait [41], face and fingerprint [24] or face and the shoulder region [23], among others.

Despite these recent works, automated gender estimation still remains a challenge and it is impacted by other soft biometrics, for example, age and ethnicity; gender dimorphism is accentuated only in adults, and variations across different ethnicities.

\section{AlgORITHM DESCRIPTION}

We show in Fig. 1 the general scheme presented in this work. Given an image of a subject, the face is detected and related appearance features are extracted and classified by a face gender estimation algorithm. We additionally estimate gender through body information already included in the same image to improve the performance. For that aim, the silhouette of the subject is extracted to first determine the distance from the subject to the camera and then predict the gender according to that specific distance setting. Later, shape and textures 
information is used in our body gender estimation approach. Finally, face and body gender estimates are combined to give an improved estimation of the gender in the three specific distance-settings.

We proceed to elaborate on the employed face and proposed body gender estimation algorithms.

\section{A. Face Gender Estimation algorithm (FGE)}
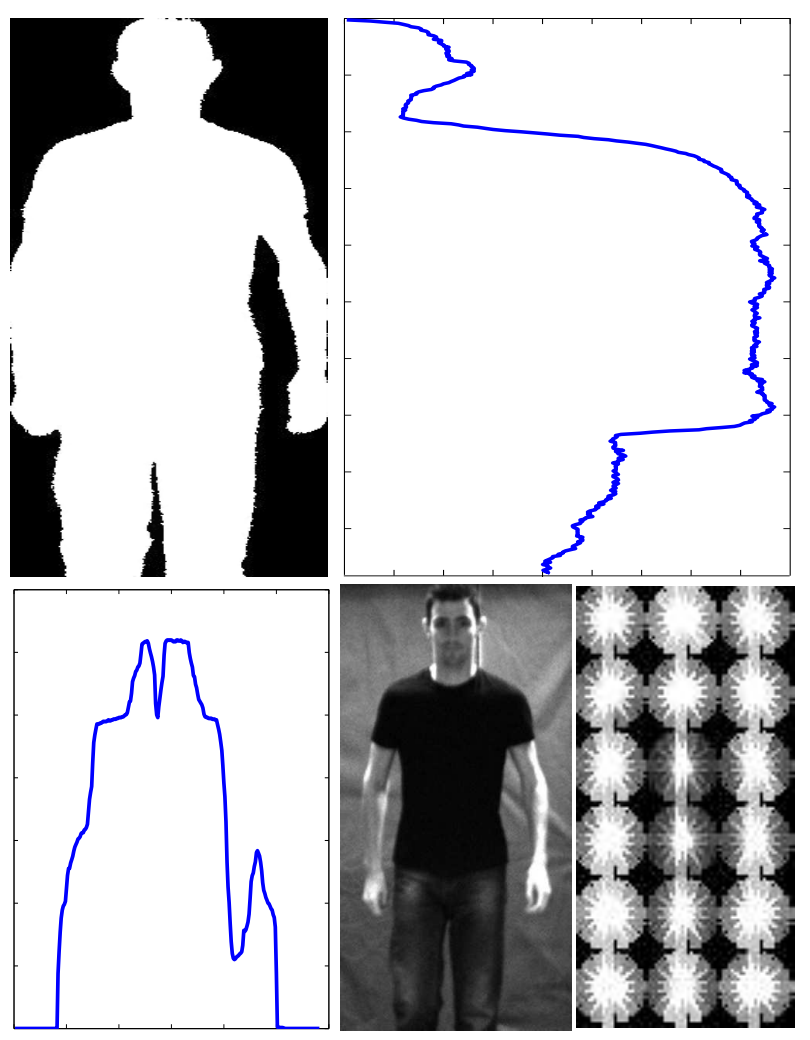

Fig. 2. Body Shape and Texture Features of the BGE algorithm proposed in this work for the far distance-setting. The sum of foreground pixels at row level are computed from top to bottom (Row Profile) while the sum of foreground pixels at column level are computed from left to right (Column Profile). HOG features are also drawn in the lower corner.

For face-based gender estimation we use OpenBR [21], a publicly available open source software for biometric recognition and evaluation. The utilized gender estimation algorithm is based on the work by Klare et al. [20]. Specifically, a face image is represented by extracting histograms of local binary pattern (LBP) and scale-invariant feature transform (SIFT) features computed on a dense grid of patches. Subsequently, the histograms from each patch are projected onto a subspace generated using PCA obtaining a feature vector. SVM is used for the final gender classification. The OpenBR gender classification algorithm has been validated on a FERET ${ }^{1}$ subset, attaining accuracies of $96.91 \%$ and $82.98 \%$ for male and female classification respectively, and an overall true classification rate of $90.57 \%$ [6], outperforming algorithms such as Neural Networks on the same dataset [27].

\footnotetext{
${ }^{1}$ http://www.nist.gov/it1/iad/ig/colorferet.cfm
}

\section{B. Body Gender Estimation algorithm (BGE)}

For body-based gender estimation we propose a novel algorithm that relies on shape-based features extracted from body silhouettes and texture information using HOG features.

1) Body Shape-based features: We here compute continuous key-measures related to body height, body width, shoulder, hips or chest from the body silhouette, rather than obtaining discrete values. By having such continuous body information, we ensure to include mentioned key body-measurements, even in unconstrained scenarios, where the extraction of individual measures is problematic.

Silhouettes are first extracted by a baseline background subtraction technique, setting the background as the initial frame of the sequence. The background subtraction is carried out in each of the $3 \mathrm{RGB}$ channels and then the RGB result is grayscaled. The binarized silhouette is obtained after thresholding the gray scale image with a global threshold. The foreground segmentation is followed by some morphological noise reduction operations, in order to delete isolated foreground areas in the image. We proceed to select the bounding box around the foreground silhouette and normalize it to $300 \times 600$ pixels. For feature extraction, we compute the sum of foreground pixels at row level from top to bottom (row profile) and the sum of foreground pixels at column level from left to right (column profile). The final body-shape feature vector constitutes the concatenation of row and column profiles. On the upper part of Fig. 2, we show the binarized image and the row (right) and column (below) profiles of a subject at the far distance-setting.

2) Texture information: Previous works predicting gender based on body information have used texture information through histogram of gradients (HOG) [5], [7]. Similarly, in our work, texture information is retained by computing HOG features from grayscale images using libraries from [13]. We use the same bounding box computed by the foreground segmentation algorithm, and then divide the resulting image into $3 \times 6$ blocks of size $100 \times 100$ pixels. We compute the histogram of gradients feature vector of each block using 8 orientations. The final HOG feature vector is the concatenation of the histograms computed per each block. Fig. 2 depicts an example of the texture features proposed in this work at the far distance-scenario.

3) Classification: Support vector machines (SVMs) with linear kernels are used as classifiers for both, body shape-based and texture algorithms. Subsequently scores of the body-shape based and texture information are fused using a score-level scheme.

We use a specific gender estimation model for each distance-setting. We distinguish between three distancesettings: far, medium and close. Although the algorithm employed is the same in all distance-settings, the gender model highly depends on the distance (far, medium and close). As a specific model is built for each distance-setting, a distancesetting classifier is required. To classify an image into one of the three distance-settings we use information based on the face-height, face-width, number of foreground pixels, as well 
as face quality (distance from the face space). All of these measures are provided by OpenBR. We classify these features by an SVM classifier with Gaussian kernel. While in images complying to the close-setting mainly the face and upper torso are visible, images complying to the far-setting expose the face and the full body of the subject. The medium-setting represents an intermediate setting between far and close distance-settings. Fig. 1 shows example images of the three distance-settings.

\section{DATABASE AND EXPERIMENTAL PROTOCOL}

Datasets traditionally used for face gender estimation include Labeled Faces in the Wild (LFW) ${ }^{2}$, MORPH $^{3}$ and Feret ${ }^{4}$. For body-based gender estimation, the Pedestrian Database from MIT [32] and VIPeR [17] databases, originally created for pedestrian detection, are commonly used. However, those databases do not have a standard experimental protocol for gender recognition and do not contain ground-truth annotations publicly available. Also, our body shape algorithm would require foreground segmentation of the images that is not provided along with those databases.

Furthermore, as one goal is to study the effect of the subjectcamera distance, we require a database that contains images of the same subjects at different distances. The Multi-Biometric Tunnel database [34] fits this requirement, as it was created to simulate a constrained environment similar to airports, ideal for automatic gait recognition. A tunnel was provided with eight cameras to acquire side and frontal images while the subject is walking through the tunnel. In this paper, only a subset of frontal images obtained from one camera is used. The frontal camera is placed at the end of the tunnel. We utilize gender ground-truth annotations, which have been introduced for previous works [39] and made publicly available.

The Multi-Biometric Tunnel database contains 222 subjects, with 150 being male and 72 female. The predominant ethnicity in the database is Caucasian (76\%), followed by Asian (14\%), Middle-East (7\%), Black (2\%) and Indian (1\%). Regarding the age, the majority of the subjects $(76.12 \%)$ are in the range of $18-28$ years; a $17.11 \%$ are in the range of $28-40$ years and only a minority are above 40 years and under 18 years.

In this work, a subset of 140 subjects $(70$ male and 70 female) among the frontal images has been selected to ensure a balanced dataset, with only 1 image per subject and distance-setting. We utilize images conforming to three distance-settings: a) far, b) medium and c) close corresponding to a) beginning of the tunnel, b) middle and c) end of the tunnel.

Experiments are carried out using a 5-fold cross validation protocol. For the body algorithm, the set of 70 subjects per gender is divided into 5 folds in order to assure a balanced number of training images ${ }^{5}$. We use all scores from all folds to compute the equal error rate (EER). With the threshold

\footnotetext{
${ }^{2}$ http://vis-www.cs.umass.edu/lfw/

${ }^{3} \mathrm{http}: / /$ faceaginggroup.com/

${ }^{4} \mathrm{http} / / /$ www.nist.gov/itl/iad/ig/colorferet.cfm

${ }^{5}$ The selected identities can be found at goo.gl/WnpNJD?gdriveurl
}

associated to the EER, overall accuracies and true positive rates (TPR) for Male and Females are computed.

\section{EXPERIMENTAL RESULTS}

\section{A. Classification of distance-settings}

Results of the distance-setting classification are reported following a leave one out protocol having 140 samples per distance. The true classification rates per distance-setting are: $97.85 \%, 98.57 \%$ and $100 \%$ for far, medium and close distance respectively.

\section{B. Face and Body Gender Estimation}

Firstly, we estimate gender by the FGE and the proposed BGE algorithms independently. Fig. 3 visually shows the results obtained for the FGE and BGE algorithms in the three distance-settings considered in red and blue colors respectively. The specific True Positive Rates (TPR) for Male and Female and overall Accuracies (Acc.) are reported in Table I.

Regarding FGE algorithm, as can be seen in Fig. 3, its overall accuracy (Acc.) improves as the distance between subject and camera decreases. This is intuitive, since both size and resolution of faces increase with a decreased distance. Specifically, a significant relative improvement of $56 \%$ is achieved - from the close $(89.28 \%)$ to the far $(57.14 \%)$ distance setting.

If we analyse in detail the FGE results through the Male TPR and Female TPR reported in Table I, we notice that in the far distance-setting, the FGE algorithm has a predominant male-categorization ( $94.28 \%$ vs. $20 \%$ ). Given the 2-class gender classification problem, we observe a poor performance of FGE in the far distance-setting. We note that the resolution of a specific subject in the far distance-setting is $82 \times 94$ pixels. For medium distances, Male and Female TPR improve, as well as the related difference reduces, obtaining an accuracy of $80.71 \%$. An improvement of accuracy was expected as faces at medium distances are of size approximately $144 \times 148$ pixels. We observe though that in the close distance-setting, TPR improve further and are very similar for both classes, probably due to the increase of resolution $(450 \times 378$ pixels for a specific subject).

It is also worth noticing the striking improvement between the far and medium scenario (from $57.14 \%$ to $80.71 \%$ ), suggesting us that the minimum resolution of faces should be bigger than $82 \times 94$ pixels. More intermediate frames will be analysed to elaborate on the minimum resolution.

Correspondingly, the accuracy of BGE decreases as the camera-subject distance decreases. The BGE algorithm performs well (above $85 \%$ of accuracy) for the far and medium distance-settings. Hence, we conclude from these results that the torso might be more discriminative, as the accuracy is stable, even if the lower part of the body (legs) is less visible in the medium distance-setting. At close distances, only the upper torso of the subject is visible and hence the accuracy drops down to $79.28 \%$ (relative decreasing of $9.75 \%$ with respect to the far distance-setting). We note that there are no 
TABLE I

Performance (\%) of the Face Gender Estimation Algorithm (FGE); the Body Gender Estimation algorithm (BGE); Sum Fusion And Smarter Sum Fusion of FGE and BGE in terms of True Positive Rate (TPR) for Male And Female (Fem.), overall Accuracy (AcC.). BEST PERFORMANCE (IN TERMS OF ACC.) OF EACH DISTANCE-SETTING IS BOLDED.

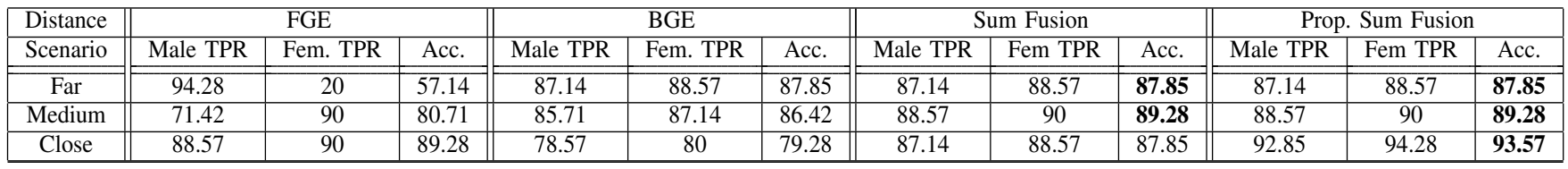

significant differences between Male and Female TPR for the BGE algorithm at any distance-setting.

As the FGE algorithm is based only on texture features, its performance drops heavily with increased distance, in which face resolution is too low to extract reliable texture features. The BGE algorithm, however, is also based on shape features, hence the performance depends more on the visibility of the body, rather than the resolution.

\section{Fusion of Face and Body}

Two different approaches of fusion have been carried out in this work: a single fusion and a weighted fusion, both at a score level. In the first approach, firstly the scores are normalized to the same range of values, then face and body scores are combined following a sum fusion. Fig. 3 shows in black and dashdot the performance of this first fusion approach. From the curve, one may notice that the fusion of the two modalities at the far distance-setting does not improve the accuracy of the best modality, in this case BGE, meaning that at that distance, the performance of the BGE algorithm is best. In the medium distance-setting, the accuracy of the fusion outperforms any of the individual modalities (face and body), achieving a relative improvement of $10 \%$ with respect to the FGE accuracy. For the close distance setting, the accuracy of the fusion drops slightly, reaching results below the accuracy of the FGE algorithm. This drop of accuracy coincides with the drop of accuracy of the BGE due to the limited body-visibility in the images in the close distance-setting. We follow with an improved fusion scheme.

The improved fusion is inspired by the proportions of the human body. From anthropometrical studies, it is known that the height of a subject is approximately 7.5 times the height of the head [16]. By exploring the ratio of the height of the face $h \_f a c e$ and the height of the body $h \_b o d y$ presented in a frame, one could estimate the body-visibility. For instance, a ratio of 0.5 indicates that the portion of the body present in the image is only 2 times the height of the head, therefore, only the upper part of the body is visible. After carrying out different experiments, we have reached improved fusion results using different weights for FGE and BGE, where the weight associated to the FGE is the ratio $h \_f a c e / h \_b o d y$ plus an offset and the weight associated to the BG estimation is the complementary to 1 . So, for the close distance-setting the FGE-weight is bigger than the BGE-weight; for the far distance-setting the FGE-weight would be smaller than the BGE-weight, and similar weights would be used for the medium distance-setting. Fig. 3 shows in cyan and dashed the performance of this improved fusion scheme, in which one may observe that the accuracy is always equal or bigger than the baseline fusion scheme. The performance in the close distance-setting is mostly improving from the proposed improved fusion, with an absolute improvement of 5.72 .

\section{COnClusions And Future Work}

In this work, we proposed a novel gender-estimation algorithm based on the fusion of extracted facial and body features. The main benefit of this approach is that, unlike face and gait multimodal approaches, the face and the body features can be extracted from one single shot image.

The proposed algorithm utilizes and complements an existing state-of-the-art gender estimation algorithm based on facial characteristics. The proposed algorithm exhibited performance improvements over both, individual face and body algorithms in scenarios where the quality of the face (in the image) was too poor to determine the gender correctly. Such scenarios can often occur in video surveillance-data.

The improvements are attributed to a novel body gender estimation algorithm that we have introduced. Unlike previous body gender estimation algorithms, our approach is the first to extract shape information from binarized images. It is also one of the few approaches that uses jointly shape and texture related features in their model. We consider our approach robust to challenging conditions thanks to the advancements of people tracking and background substraction techniques.

We additionally analysed the impact of distance between subject and camera, investigating different distance-settings, that range from full body exposure to head and upper torso visibility. The analysis showed that the body gender estimation algorithm (BGE) performs reasonably well when a large part of the body is visible, while the face gender estimation algorithm (FGE) is robust only at close distances.

Future work will test the proposed algorithm on less constrained databases that include pose variations. Further, we will explore other features recently employed for body-based people identification such as contour coordinates [15] and will study more sophisticated fusion schemes [14].

\section{ACKNOWLEDGMENT}

This work has been partially supported by projects CogniMetrics (TEC2015-70627-R) from Spanish MINECO. E. Gonzalez-Sosa was supported by a PhD scholarship from Universidad Autonoma de Madrid. A. Dantcheva was supported 


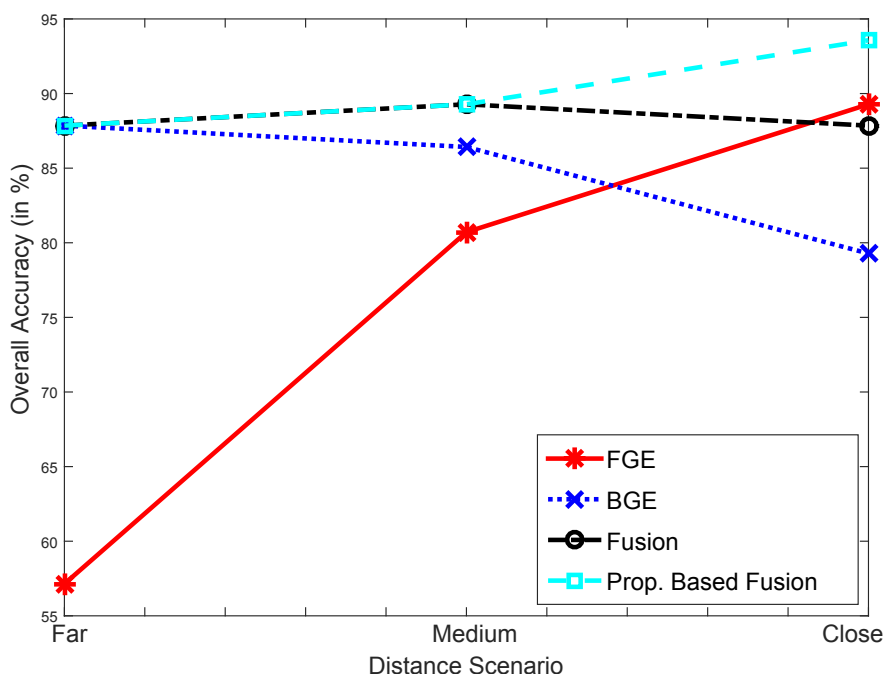

Fig. 3. Resuls obtained for the Face Gender Estimation Algorithm (FGE); Body Gender Estimation Algorithm (BGE); and the fusion of FGE and BGE following two different schemes. Results are reported in terms of Overall Accuracy ( in \%).

by the French Government (National Research Agency, ANR) through the "Investments for the Future" Program reference \#ANR-11-LABX-0031-01.

\section{REFERENCES}

[1] G. Antipov, S.-A. Berrani, N. Ruchaud, and J.-L. Dugelay. Learned vs. hand-crafted features for pedestrian gender recognition. In Proc. of ACM Int. Conf. on MM, pages 1263-1266, 2015.

[2] J. Bekios-Calfa, J. M. Buenaposada, and L. Baumela. Revisiting linear discriminant techniques in gender recognition. IEEE Trans. on PAMI, 33(4):858-864, 2011.

[3] J. Bekios-Calfa, J. M. Buenaposada, and L. Baumela. Robust gender recognition by exploiting facial attributes dependencies. Pattern Recognition Letters, 2013.

[4] P. Bilinski, A. Dantcheva, and F. Bremond. Can a smile reveal your gender? In IEEE Proc. of BIOSIG, 2016.

[5] L. Cao, M. Dikmen, Y. Fu, and T. S. Huang. Gender recognition from body. In Proc. of ACM Int. Conf. on MM, 2008.

[6] C. Chen, A. Dantcheva, and A. Ross. Impact of facial cosmetics on automatic gender and age estimation algorithms. In Proc. of VISAPP, 2014.

[7] M. Collins, J. Zhang, P. Miller, and H. Wang. Full body image feature representations for gender profiling. In IEEE Proc. of ICCVW, pages $1235-1242,2009$.

[8] T. E. Currie and A. C. Little. The relative importance of the face and body in judgments of human physical attractiveness. Evolution and human behavior, 30(6):409-416, 2009.

[9] A. Dantcheva and F. Bremond. Gender estimation based on smiledynamics. IEEE Trans. on IFS, 2016.

[10] A. Dantcheva, P. Elia, and A. Ross. What else does your biometric data reveal? a survey on soft biometrics. IEEE Trans. on IFS, 11(3):441-467, March 2016.

[11] A. Dantcheva, A. Singh, P. Elia, and J.-L. Dugelay. Search pruning in video surveillance systems: Efficiency-reliability tradeoff. In IEEE Proc. of ICCVW, pages 1356-1363, 2011.

[12] A. Dantcheva, C. Velardo, A. Dangelo, and J.-L. Dugelay. Bag of soft biometrics for person identification. Multimedia Tools and Applications, 51(2):739-777, 2011.

[13] P. Dollár. Piotrs computer vision matlab toolbox (pmt). http:/vision ucsd edu/ pdollar/toolbox/doc/index.html, 2014.

[14] J. Fierrez-Aguilar, J. Ortega-Garcia, J. Gonzalez-Rodriguez, and J. Bigun. Discriminative multimodal biometric authentication based on quality measures. Pattern Recognition, 38(5):777-779, 2005.
[15] E. Gonzalez-Sosa, R. Vera-Rodriguez, J. Fierrez, M. Moreno-Moreno, and J. Ortega-Garcia. Feature exploration for biometric recognition using millimetre wave body images. EURASIP Journal on Image and Video Processing, 2015(1):1-13, 2015.

[16] C. M. Goss. Gray's anatomy of the human body. Academic Medicine, 35(1):90, 1960.

[17] D. Gray, S. Brennan, and H. Tao. Evaluating appearance models for recognition, reacquisition, and tracking. In IEEE Proc. of PETS), volume 3. Citeseer, 2007.

[18] G. Guo, G. Mu, and Y. Fu. Gender from body: A biologically-inspired approach with manifold learning. In Computer Vision, pages 236-245. Springer, 2010.

[19] A. K. Jain, S. C. Dass, and K. Nandakumar. Soft biometric traits for personal recognition systems. In Biometric Authentication, pages 731738. Springer, 2004.

[20] B. F. Klare, M. J. Burge, J. C. Klontz, R. W. V. Bruegge, and A. K. Jain. Face recognition performance: Role of demographic information. IEEE Trans. on IFS, 7(6):1789-1801, 2012.

[21] J. C. Klontz, B. F. Klare, S. Klum, A. K. Jain, and M. J. Burge. Open source biometric recognition. In IEEE Proc. of BTAS, pages 1-8. IEEE, 2013.

[22] J. H. Langlois and L. A. Roggman. Attractive faces are only average Psychological science, 1(2):115-121, 1990.

[23] M. Li, S. Bao, W. Dong, Y. Wang, and Z. Su. Head-shoulder based gender recognition. In IEEE Proc. of ICIP, pages 2753-2756, 2013.

[24] X. Li, X. Zhao, Y. Fu, and Y. Liu. Bimodal gender recognition from face and fingerprint. In IEEE Proc. of CVPR, pages 2590-2597, 2010.

[25] T. Linder, S. Wehner, and K. O. Arras. Real-time full-body human gender recognition in (rgb)-d data. In IEEE Proc. of ICRA, pages 3039 3045, 2015.

[26] E. Mäkinen and R. Raisamo. Evaluation of gender classification methods with automatically detected and aligned faces. IEEE Trans. on PAMI, 30(3):541-547, 2008.

[27] E. Mäkinen and R. Raisamo. Evaluation of gender classification methods with automatically detected and aligned faces. IEEE Trans. on PAMI, 30(3):541-547, 2008.

[28] A. Nambiar, A. Bernardino, and J. Nascimento. Shape context for soft biometrics in person re-identification and database retrieval. Pattern Recognition Letters, 68:297-305, 2015.

[29] C. B. Ng, Y. H. Tay, and B.-M. Goi. Vision-based human gender recognition: A survey. PRICAI Trends in Artificial Intelligence, 7458:335-346, 2012.

[30] K. Niinuma, U. Park, and A. K. Jain. Soft biometric traits for continuous user authentication. IEEE Trans. on IFS, 5(4):771-780, 2010.

[31] M. S. Nixon, P. L. Correia, K. Nasrollahi, T. B. Moeslund, A. Hadid, and M. Tistarelli. On soft biometrics. Pattern Recognition Letters, 68 Part 2:218 - 230, 2015. Special Issue on Soft Biometrics.

[32] M. Oren, C. Papageorgiou, P. Sinha, E. Osuna, and T. Poggio. Pedestrian detection using wavelet templates. In IEEE Proc. of CVPR, pages 193199, 1997.

[33] D. Reid, S. Samangooei, C. Chen, M. Nixon, and A. Ross. Soft biometrics for surveillance: an overview. Machine learning: theory and applications, pages 327-352, 2013.

[34] R. D. Seely, S. Samangooei, M. Lee, J. N. Carter, and M. S. Nixon. The university of southampton multi-biometric tunnel and introducing a novel 3d gait dataset. In IEEE Proc. of BTAS, pages 1-6, 2008.

[35] C. Shan, S. Gong, and P. W. McOwan. Learning gender from human gaits and faces. In IEEE Proc. of AVVS, pages 505-510, 2007.

[36] C. Shan, S. Gong, and P. W. McOwan. Fusing gait and face cues for human gender recognition. Neurocomputing, 71(10):1931-1938, 2008.

[37] V. Sorokin and I. Makarov. Gender recognition from vocal source. Acoustical Physics, 54(4):571-578, 2008.

[38] M. Toews and T. Arbel. Detection, localization, and sex classification of faces from arbitrary viewpoints and under occlusion. IEEE Trans. on PAMI, 31(9):15671781, 2009.

[39] P. Tome, J. Fierrez, R. Vera-Rodriguez, and M. S. Nixon. Soft biometrics and their application in person recognition at a distance. IEEE Trans. on TIFS, 9(3):464-475, 2014.

[40] J. Wang, J. Li, W. Yau, and E. Sung. Boosting dense sift descriptors and shape contexts of face images for gender recognition. In Proc. of CVPRW, page 96172, 2010.

[41] D. Zhang and Y.-H. Wang. Gender recognition based on fusion on face and gait information. In IEEE Proc. of ICMLC, volume 1, pages 62-67, 2008 . 